TRANSACTIONS OF THE

AMERICAN MATHEMATICAL SOCIETY

Volume 356, Number 4, Pages 1489-1499

S 0002-9947(03)03458-5

Article electronically published on October 29, 2003

\title{
ANALYTIC $p$-ADIC CELL DECOMPOSITION AND INTEGRALS
}

\author{
RAF CLUCKERS
}

\begin{abstract}
We prove a conjecture of Denef on parameterized $p$-adic analytic integrals using an analytic cell decomposition theorem, which we also prove in this paper. This cell decomposition theorem describes piecewise the valuation of analytic functions (and more generally of subanalytic functions), the pieces being geometrically simple sets, called cells. We also classify subanalytic sets up to subanalytic bijection.
\end{abstract}

\section{INTRODUCTION}

Let $p$ denote a fixed prime number, $\mathbb{Z}_{p}$ the ring of $p$-adic integers, $\mathbb{Q}_{p}$ the field of $p$-adic numbers, $|\cdot|$ the $p$-adic norm, and $v(\cdot)$ the $p$-adic valuation.

Let $f=\left(f_{1}, \ldots, f_{r}\right)$ be an $r$-tuple of restricted power series over $\mathbb{Z}_{p}$ in the variables $(\lambda, x)=\left(\lambda_{1}, \ldots, \lambda_{s}, x_{1}, \ldots, x_{m}\right)$, i.e., the $f_{i}$ are power series converging on $\mathbb{Z}_{p}^{s+m}$. To $f$ we associate a parametrized $p$-adic integral

$$
I(\lambda)=\int_{\mathbb{Z}_{p}^{m}}|f(\lambda, x)||d x|,
$$

where $|d x|$ is the Haar measure on $\mathbb{Z}_{p}^{m}$ normalized so that $\mathbb{Z}_{p}^{m}$ has measure 1.

A subanalytic constructible function on a subanalytic set $X$ is by definition a $\mathbb{Q}$-linear combination of products of functions of the form $v(h)$ and $\left|h^{\prime}\right|$, where $h: X \rightarrow \mathbb{Q}_{p}^{\times}$and $h^{\prime}: X \rightarrow \mathbb{Q}_{p}$ are subanalytic functions. (For the notion of subanalytic functions and subanalytic sets we refer to the section Terminology and notation below.)

We prove the following conjecture of Denef [8]:

Theorem 1.1. The function I is a subanalytic constructible function on $\mathbb{Z}_{p}^{s}$.

In the case that the functions $f_{i}$ are polynomials, the map $I$ has been studied by Igusa for $r=1$, by Lichtin for $r=2$, and by Denef for arbitrary $r$ (see [16, 17, 18], [19], and [8]). In the more general case that the $|f(\lambda, x)|$ in (1) is replaced by an arbitrary subanalytic constructible function, the conclusion of Theorem 1.1 still holds (see Theorem 4.2 below), where now $I$ is identically zero if the integrated function is not integrable for some $\lambda$.

Received by the editors August 15, 2002.

2000 Mathematics Subject Classification. Primary 11S80, 32P05, 32B20; Secondary 03C10, 03C98, 11U09, 11S40.

Key words and phrases. Subanalytic $p$-adic sets, cell decomposition, $p$-adic integrals, Igusa's local zeta functions.

The author is a Research Assistant of the Fund for Scientific Research - Flanders (Belgium)(F.W.O.). 
The rationality of the analytic $p$-adic Serre-Poincaré series was conjectured in [26] and [27] and proven by Denef and van den Dries in [9]; the rationality can immediately be obtained as a corollary of integration Theorem 1.1. This is because it is well known how to express the Poincaré series as a $p$-adic integral (see [8], section 1.6).

A second key result of the present paper is a cell decomposition theorem for subanalytic sets and subanalytic functions (Theorem 2.3), in perfect analogy to the semialgebraic cell decomposition theorem of [4] and [6]. Roughly speaking, $p$-adic cell decomposition theorems describe the norm of given functions after partitioning the domain of the functions in finitely many basic sets, called cells. Cell decompositions are very useful to study parameterized $p$-adic integrals (see below and [8]) and to prove the rationality of Igusa's local zeta functions and of several Poincaré series (see [4]). Many of the applications of cell decomposition (in for example [8] and [5]) cannot, up to now, be proven with other techniques.

The proof of the analytic cell decomposition is based on several results by van den Dries, Haskell, and Macpherson [13] on the geometry of subanalytic $p$-adic sets and subanalytic functions; we state some of these results in section 2 .

We also extensively use the theory of $p$-adic subanalytic sets, developed by Denef and van den Dries in [9] in analogy to the theory of real subanalytic sets; in particular, we use the dimension theory of [9]. In section 3 we apply cell decomposition to obtain the following classification:

Theorem 1.2. Let $X \subset \mathbb{Q}_{p}^{m}$ and $Y \subset \mathbb{Q}_{p}^{n}$ be infinite subanalytic sets. Then there exists a subanalytic bijection $X \rightarrow Y$ if and only if $\operatorname{dim}(X)=\operatorname{dim}(Y)$.

This classification of subanalytic sets is similar to the classification of semialgebraic sets in 2]. Note that in particular there exists a semialgebraic bijection between $\mathbb{Q}_{p}$ and $\mathbb{Q}_{p}^{\times}$; this is the main result of [3].

The theory of $p$-adic integration has also served as an inspiring example for the theory of motivic integration and there are many connections to it (see e.g. [11] and [10]).

Many of the results of [9] and [13] are formulated for $\mathbb{Q}_{p}$ and not for finite field extensions of $\mathbb{Q}_{p}$; nevertheless, all results referred to in this paper also hold for finite field extensions of $\mathbb{Q}_{p}$ (see the remark in (3.31) of [9]). All results of this paper also hold in finite field extensions of $\mathbb{Q}_{p}$.

Terminology and notation. Let $p$ denote a fixed prime number, $\mathbb{Q}_{p}$ the field of $p$-adic numbers and $K$ a fixed finite field extension of $\mathbb{Q}_{p}$ with valuation ring $R$. For $x \in K^{\times}$let $v(x) \in \mathbb{Z}$ denote the $p$-adic valuation of $x$ and $|x|=q^{-v(x)}$ the $p$-adic norm, with $q$ the cardinality of the residue class field. We write $P_{n}=\left\{y^{n} \mid y \in K^{\times}\right\}$, and $\mu P_{n}$ denotes $\left\{\mu x \mid x \in P_{n}\right\}$ for $\mu \in K$.

For $x=\left(x_{1}, \ldots, x_{m}\right)$ let $K\{x\}$ be the ring of restricted power series over $K$ in the variables $x$; it is the ring of power series $\sum a_{i} x^{i}$ in $K[[x]]$ such that $\left|a_{i}\right|$ tends to 0 as $|i| \rightarrow \infty$. (Here, we use the multi-index notation where $i=\left(i_{1}, \ldots, i_{m}\right)$, $|i|=i_{1}+\ldots+i_{m}$ and $x^{i}=x_{1}^{i_{1}} \ldots x_{m}^{i_{m}}$.) For $x_{0} \in R^{m}$ and $f=\sum a_{i} x^{i}$ in $K\{x\}$ the series $\sum a_{i} x_{0}^{i}$ converges to a limit in $K$, thus, one can associate to $f$ a restricted analytic function given by

$$
f: K^{m} \rightarrow K: x \mapsto \begin{cases}\sum_{i} a_{i} x^{i} & \text { if } x \in R^{m} \\ 0 & \text { else. }\end{cases}
$$


We extend the notion of $D$-functions of [9] to our setting:1

Definition 1.3. A $D$-function is a function $K^{m} \rightarrow K$ for some $m \geq 0$, obtained by repeated application of the following rules:

(i) for each $f \in K\left\{x_{1}, \ldots, x_{m}\right\}$, the associated restricted analytic function $x \mapsto f(x)$ is a $D$-function;

(ii) for each polynomial $f \in K\left[x_{1}, \ldots, x_{m}\right]$, the polynomial map $x \mapsto f(x)$ is a $D$-function;

(iii) the function $x \mapsto x^{-1}$, where $0^{-1}=0$ by convention, is a $D$-function;

(iv) for each $D$-function $f$ in $n$ variables and each $D$-functions $g_{1}, \ldots, g_{n}$ in $m$ variables, the function $f\left(g_{1}, \ldots, g_{n}\right)$ is a $D$-function.

A (globally) subanalytic subset of $K^{m}$ is a subset of the form

$$
X=\bigcup_{i=1}^{r} \bigcap_{j=1}^{s} X_{i j},
$$

where each $X_{i j}$ is of the form $\left\{x \in K^{m} \mid f_{i j}(x)=0\right\}$ or $\left\{x \in K^{m} \mid f_{i j}(x) \in P_{n_{i j}}\right\}$, where the functions $f_{i j}$ are $D$-functions and $n_{i j}>0$. We call a function $g: A \subset$ $K^{m} \rightarrow K^{n}$ subanalytic if its graph is a subanalytic set. We refer to [9], [8], and 13. for the theory of subanalytic $p$-adic geometry and to [21] for the theory of rigid subanalytic sets.

In section 2 we will use the framework of model theory. We let $\mathcal{L}_{\text {an }}$ be the first order language consisting of the symbols

$$
+,-, \cdot,^{-1},\left\{P_{n}\right\}_{n>0},
$$

together with an extra function symbol $f$ for each restricted analytic function associated to restricted power series in $\bigcup_{m} K\left\{x_{1}, \ldots, x_{m}\right\}$. We consider $K$ as an $\mathcal{L}_{\text {an }}$-structure using the natural interpretations of the symbols of $\mathcal{L}_{\text {an }}$.

We mention the following fundamental result in the theory of subanalytic sets.

Theorem 1.4 ([9], Corollary (1.6)). The collection of subanalytic sets is closed under taking complements, finite unions, finite intersections, and images under subanalytic maps 2

A semialgebraic subset of $K^{m}$ is a subset of the same form as $X$ above but with the $f_{i j}$ polynomials over $K$, and a function is semialgebraic if its graph is a semialgebraic set. It is well known that also the collection of semialgebraic sets is closed under taking complements, finite unions and intersections, and images under semialgebraic maps (see [23, [6]).

\section{AnAlytic CELl DeComposition}

To state cell decomposition one needs basic sets called (subanalytic) cells, which we define inductively. For $m, l>0$ write $\pi_{m}: K^{m+l} \rightarrow K^{m}$ for the linear projection on the first $m$ variables and, for $A \subset K^{m+l}$ and $x \in \pi_{m}(A)$, write $A_{x}$ for the fiber $\left\{t \in K^{l} \mid(x, t) \in A\right\}$.

\footnotetext{
${ }^{1}$ In [9], $D$-functions are functions from $R^{m}$ to $R$ for $m \geq 0$.

${ }^{2}$ I take the occasion to correct a small error in [13] with respect to quantifier elimination on $\mathbb{Q}_{p}$. Namely, the division function $D$ in [13] should either be replaced by the field inverse ${ }^{-1}$ or by the function $D$ given by $D(x, y)=x / y$ if $0<|x| \leq|y|$ and $D(x, y)=0$ otherwise.
} 
Definition 2.1. A cell $A \subset K$ is a (nonempty) set of the form

$$
\left\{t \in K|| \alpha\left|\square_{1}\right| t-\gamma\left|\square_{2}\right| \beta \mid, t-\gamma \in \mu P_{n}\right\},
$$

with constants $n>0, \mu, \gamma \in K, \alpha, \beta \in K^{\times}$, and $\square_{i}$ either $<$ or no condition. If $\mu=0$ we call $A$ a 0 -cell and we call $A$ a 1-cell otherwise.

A (subanalytic) cell $A \subset K^{m+1}, m \geq 1$, is a set of the form

$$
\begin{aligned}
\left\{(x, t) \in K^{m+1} \mid\right. & x \in D,|\alpha(x)| \square_{1}|t-\gamma(x)| \square_{2}|\beta(x)|, \\
& \left.t-\gamma(x) \in \mu P_{n}\right\},
\end{aligned}
$$

with $(x, t)=\left(x_{1}, \ldots, x_{m}, t\right), n>0, \mu \in K, D=\pi_{m}(A)$ a cell, subanalytic functions $\alpha, \beta: K^{m} \rightarrow K^{\times}, \gamma: K^{m} \rightarrow K$, and $\square_{i}$ either $<$ or no condition. We call $\gamma$ the center and $\mu P_{n}$ the coset of the cell $A$. If $D$ is a cell of type $\left(i_{1}, \ldots, i_{m}\right)$ with $i_{j} \in\{0,1\}$, we call $A$ an $\left(i_{1}, \ldots, i_{m}, 0\right)$-cell if $\mu=0$ and we call $A$ an $\left(i_{1}, \ldots, i_{m}, 1\right)$ cell otherwise. If at each stage of this inductive definition all occurring functions $\alpha, \beta$, and $\gamma$ are analytic on the respective projections $\pi_{i}(A), i=1, \ldots, m-1$, we call $A$ an analytic cell.

Remark 2.2. (i) An $\left(i_{1}, \ldots, i_{m}, 0\right)$-cell $A \subset K^{m+1}$ is the graph of a subanalytic function defined on $\pi_{m}(A)$, and, if $A$ is an $\left(i_{1}, \ldots, i_{m}, 1\right)$-cell, then $A_{x}$ is a nonempty open in $K$ for each $x \in \pi_{m}(A)$.

(ii) An analytic cell is a $K$-analytic manifold (in the obvious sense, see e.g. [1).

Theorem 2.3 below is a subanalytic analogue of the semialgebraic cell decomposition (see [4] and [6]); it is a perfect analogue of the reformulation [2] Lemma 4]. In [8, an overview is given of applications of $p$-adic cell decomposition, going from a description of local singular series to counting profinite $p$-groups (as in 14]).

Theorem 2.3 (Analytic Cell Decomposition). Let $X \subset K^{m+1}$ be a subanalytic set, $m \geq 0$, and $f_{j}: X \rightarrow K$ subanalytic functions for $j=1, \ldots, r$. Then there exists a finite partition of $X$ into cells $A$ with center $\gamma: K^{m} \rightarrow K$ and coset $\mu P_{n}$ such that for each $(x, t) \in A$

$$
\left|f_{j}(x, t)\right|=\left|\delta_{j}(x)\right|\left|(t-\gamma(x))^{a_{j}} \mu^{-a_{j}}\right|^{\frac{1}{n}}, \quad \text { for each } j=1, \ldots, r,
$$

with $(x, t)=\left(x_{1}, \ldots, x_{m}, t\right)$, integers $a_{j}$, and $\delta_{j}: K^{m} \rightarrow K$ subanalytic functions. If $\mu=0$, we use the conventions $a_{j}=0$ and $0^{0}=1$. Moreover, the cells $A$ can be taken to be analytic cells such that the $\delta_{j}$ are analytic on $\pi_{m}(A)$.

Remark 2.4. (i) Theorem 2.3 can be seen as a $p$-adic analogue of the preparation theorem [20] for real subanalytic functions, or as an analogue of cell decomposition for real subanalytic sets (see e.g. [12]).

(ii) Some of the analytic analogues of applications in 8 as well as some of the results of [5], 13] and 9] can be obtained immediately using cell decomposition and the integration theorems of this paper. For example, see Corollary 1.8.2 of 5] on local singular series, Theorem 3.1 of [8, the $p$-adic Lojasiewicz inequalities (3.37), the subanalytic selection theorem (3.6), the stratification theorem (3.29), and Theorem (3.2) of 9 . However, note that the presented proof of Theorem 2.3 relies on 9 and 13 .

(iii) Partial results towards subanalytic cell decomposition have been obtained in [22], 24], [25] and [28]. In [24] and [28], a partitioning of arbitrary subanalytic sets into cells is obtained, but without the description of the norm of subanalytic functions on these cells. However, the description of the norm of the subanalytic functions in Theorem 2.3 is used in the proof of the conjecture of Denef below. 
For the proof of Theorem 2.3 we use techniques from model theory, namely a compactness argument. (For general notions of model theory we refer to [15].)

Let $K_{1}$ be an $\mathcal{L}_{\mathrm{a} n}$-elementary extension of $K$ and let $R_{1}$ be its valuation ring. In view of Theorem 1.4, we can call a set $X \subset K_{1}^{m}$ subanalytic if it is $\mathcal{L}_{\text {an }}$-definable (with parameters from $K_{1}$ ) and analogously for subanalytic functions, cells, and so on. Expressions of the form $|x|<|y|$ for $x, y \in K_{1}$ are abbreviations for the corresponding $\mathcal{L}_{\mathrm{a} n}$-formula's expressing $|x|<|y|$ for $x, y \in K$, as in Lemma 2.1 of [6]. 3 Cells in $K_{1}^{m}$ are defined just as in $K^{m}$ by replacing $K$ by $K_{1}$ everywhere in the definition. By a $D$-function $K_{1}^{m} \rightarrow K_{1}$ we mean a function given by an $\mathcal{L}_{\text {an }}$-term (with parameters from $K_{1}$ ) in $m$ variables. Similarly, one can speak of semialgebraic subsets of $K_{1}^{m}$ (with parameters from $K_{1}$ ) 4

We recall one of the main results of [13]:

Theorem 2.5 (Theorem B of [13]). Each subanalytic subset of $K_{1}$ is semialgebraic.

The following two lemmas treat the one-dimensional part of Theorem 2.3.

Lemma 2.6. Let $f: R_{1} \rightarrow K_{1}$ be a subanalytic function. Then there exists a finite partition of $R_{1}$ into semialgebraic sets $A$ such that for each $A$ there exist polynomials $p$ and $q$ such that

$$
|f(x)|=|p(x) / q(x)|^{1 / e}, \text { for each } x \in A,
$$

where $q$ has no zeros in $A$ and $e>0$ is an integer.

Proof. By Theorem 3.6 of [7], there exists a finite partition of $R_{1}$ into subanalytic sets $B$ such that

$$
|f(x)|=\left|g_{B}(x) / h_{B}(x)\right|^{1 / e}, \text { for each } x \in B,
$$

where $g_{B}$ and $h_{B}$ are $D$-functions, $h_{B}(x) \neq 0$ on $B$ and $e>0$. (In [7] this is proven for subanalytic functions $\mathbb{Z}_{p}^{m} \rightarrow \mathbb{Z}_{p}$ using quantifier elimination in an elementary way; its proof can be repeated for our situation $R_{1} \rightarrow K_{1}$ or otherwise one can instantiate parameters in the result of [7] to deduce this as a corollary.) By Theorem B of [13], the sets $B$ are semialgebraic.

In [13] it is proven that the norm of any $D$-function in one variable is piecewise equal to the norm of a rational function, the pieces being semialgebraic sets. More precisely, by Proposition 4.1, Corollary 3.4 and Lemma 2.10 of [13], there exists for each function $g_{B}$ a finite partition of $R_{1}$ into semialgebraic sets $C$ such that on each $C$

$$
\left|g_{B}(x)\right|=\left|g_{B C}(x) / h_{B C}(x)\right|, \text { for each } x \in C,
$$

where $g_{B C}$ and $h_{B C}$ are polynomials over $K_{1}$ and $h_{B C}(x) \neq 0$ on $C$. The same holds for each function $h_{B}$. Taking an appropriate partition using intersections of these sets $C$ and $B$ the lemma follows.

Lemma 2.7. Let $X \subset R_{1}$ be a subanalytic set and $f: X \rightarrow K_{1}$ a subanalytic function. Then there exists a finite partition $\mathcal{P}$ of $X$ into cells, such that for each cell $A \in \mathcal{P}$ with center $\gamma \in K_{1}$ and coset $\mu P_{n}$

$$
|f(t)|=|\delta|\left|(t-\gamma)^{a} \mu^{-a}\right|^{\frac{1}{n}} \text { for each } t \in A,
$$

\footnotetext{
${ }^{3}$ For example, if $K=\mathbb{Q}_{p}$ with $p \neq 2$, the property $|x|<|y|$ is equivalent to $y^{2}+\frac{x^{2}}{p} \in P_{2}$.

${ }^{4}$ This can be done using the language of Macintyre, consisting of $+,-, \cdot, 0,1$, and the collection of predicates $\left\{P_{n}\right\}$ for $n>0$.
} 
with $\delta \in K_{1}$ and $a$ an integer. We use the convention that $a=0$ and $0^{0}=1$ when $\mu=0$.

Proof. We extend $f$ to a function $R_{1} \rightarrow K_{1}$ by putting $f(x)=0$ if $x \notin X$. By Theorem B of [13], the set $X$ is semialgebraic. Apply Lemma 2.6 to $f$ to obtain a partition $\mathcal{P}$. Intersecting each set in $\mathcal{P}$ with $X$, we obtain a partition $\mathcal{P}^{\prime}$ of $X$. Now apply the semialgebraic cell decomposition (in the formulation of [2 Lem. 4]) to the sets in $\mathcal{P}^{\prime}$ and the respective polynomials occurring in the application of Lemma 2.6. If we refine the obtained partition such that for each cell $A \subset C$ with coset $\mu P_{n}$ the number $n$ is a multiple of $e$ (for the occurring fractional powers $1 / e$ ), then the lemma follows.

We will use the previous lemma and a model-theoretical compactness argument to prove the following variant of Theorem 2.3 .

Theorem 2.8. Let $K_{1}$ be an arbitrary $\mathcal{L}_{\text {an }}$-elementary extension of $K$ with valuation ring $R_{1}$. Let $X \subset K_{1}^{m+1}$ be subanalytic and $f_{j}: X \rightarrow K_{1}$ subanalytic functions for $j=1, \ldots, r$. Then there exists a finite partition of $X$ into subanalytic cells $A$ with center $\gamma: K_{1}^{m} \rightarrow K_{1}$ and coset $\mu P_{n}$ such that for each $(x, t) \in A$

$$
\left|f_{j}(x, t)\right|=\left|\delta_{j}(x)\right|\left|(t-\gamma(x))^{a_{j}} \mu^{-a_{j}}\right|^{\frac{1}{n}}
$$

with $(x, t)=\left(x_{1}, \ldots, x_{m}, t\right)$, integers $a_{j}$, and $\delta_{j}: K_{1}^{m} \rightarrow K_{1}$ subanalytic functions, $j=1, \ldots, r$. Here we use the convention that $a_{j}=0$ and $0^{0}=1$ when $\mu=0$.

Proof. The proof goes by induction on $m \geq 0$. It is enough to prove the theorem for $r=1$ (the theorem then follows after a straightforward further partitioning; see for example [6]).

When $m=0$, the usual change of variables $t^{\prime}=1 / t$ reduces the description of what happens outside $R_{1}$ to what happens on $R_{1}$, and an application of Lemma 2.7 gives the desired result.

Let $X \subset K_{1}^{m+1}$ and $f: X \rightarrow K_{1}$ be subanalytic, $m>0$. We write $(x, t)=$ $\left(x_{1}, \ldots, x_{m}, t\right)$ and know by the previous discussion that for each fixed $x \in K_{1}^{m}$ we can decompose the fiber $X_{x}$ and the function $t \mapsto f(x, t)$ on this fiber. We will measure the complexity of given decompositions on which $|f(x, \cdot)|$ has a nice description and see that this must be uniformly bounded when $x$ varies.

To do this, we define a countable set $\mathcal{S}=\left\{\mu P_{n} \mid \mu \in K, n>0\right\} \times \mathbb{Z} \times\{<, \emptyset\}^{2}$ and $\mathcal{S}^{\prime}=\left(K_{1}^{\times}\right)^{2} \times K_{1}^{2}$. To each $d=\left(\mu P_{n}, a, \square_{1}, \square_{2}\right)$ in $\mathcal{S}$ and $\xi=\left(\xi_{1}, \xi_{2}, \xi_{3}, \xi_{4}\right) \in \mathcal{S}^{\prime}$ we associate a set $\operatorname{Dom}_{(d, \xi)}$ as follows:

$$
\operatorname{Dom}_{(d, \xi)}=\left\{t \in K_{1}|| \xi_{1}\left|\square_{1}\right| t-\xi_{3}\left|\square_{2}\right| \xi_{2} \mid, t-\xi_{3} \in \mu P_{n}\right\} .
$$

The set $\operatorname{Dom}_{(d, \xi)}$ is either empty or a cell and is independent of $\xi_{4}$ and $a$. For fixed $k>0$ and tuple $d=\left(d_{1}, \ldots, d_{k}\right) \in \mathcal{S}^{k}$, let $\varphi_{(d, k)}(x, \xi)$ be an $\mathcal{L}_{\text {an }}$-formula in the free variables $x=\left(x_{1}, \ldots, x_{m}\right)$ and $\xi=\left(\xi_{1}, \ldots, \xi_{k}\right)$, with $\xi_{i}=\left(\xi_{i 1}, \xi_{i 2}, \xi_{i 3}, \xi_{i 4}\right)$, such that $(x, \xi) \in K_{1}^{m+4 k}$ satisfies $\varphi_{(d, k)}$ if and only if the following are true:

(i) $x \in \pi_{m}(X)$ and $\xi \in\left(\mathcal{S}^{\prime}\right)^{k}$,

(ii) the collection of the sets $\operatorname{Dom}_{\left(d_{i}, \xi_{i}\right)}$ for $i=1, \ldots, k$ forms a partition of the fiber $X_{x}=\left\{t \in K_{1} \mid(x, t) \in X\right\}$,

(iii) $\left|\xi_{i 4}\right|\left|\left(t-\xi_{i 3}\right)^{a_{i}} \mu_{i}^{-a_{i}}\right|^{\frac{1}{n_{i}}}=|f(x, t)|$ for each $t \in \operatorname{Dom}_{\left(d_{i}, \xi_{i}\right)}$ and each $i=$ $1, \ldots, k$. 
Now we define for each $k>0$ and $d \in \mathcal{S}^{k}$ the set

$$
B_{d}=\left\{x \in K_{1}^{m} \mid \exists \xi \quad \varphi_{d}(x, \xi)\right\} .
$$

Each set $B_{d}$ is subanalytic and the (countable) collection $\left\{B_{d}\right\}_{k, d}$ covers $\pi_{m}(X)$, because each $x \in \pi_{m}(X)$ is in some $B_{d}$ by the induction. Since $K_{1}$ is an arbitrary elementary extension of $K$, finitely many sets of the form $B_{d}$ must already cover $\pi_{m}(X)$ by model-theoretical compactness. Consequently, we can take subanalytic sets $D_{1}, \ldots, D_{s}$ such that $\left\{D_{i}\right\}$ forms a partition of $\pi_{m}(X)$ and each $D_{i}$ is contained in a set $B_{d}$ for some $k>0$ and $k$-tuple $d$. For each $i=1, \ldots, s$, fix such a $d$ with $D_{i} \subset B_{d}$, and let $\Gamma_{i}$ be the subanalytic set

$$
\Gamma_{i}=\left\{(x, \xi) \in D_{i} \times\left(\mathcal{S}^{\prime}\right)^{k} \mid \varphi_{d}(x, \xi)\right\} .
$$

Then $\pi_{m}\left(\Gamma_{i}\right)=D_{i}$ by construction ( $\pi_{m}$ is the projection on the $x$-coordinates). By Theorem 3.6 of [9] on definable Skolem functions, there is a subanalytic function $D_{i} \rightarrow K_{1}^{4 k}$ associating to $x$ a tuple $\xi(x) \in\left(\mathcal{S}^{\prime}\right)^{k}$ such that $(x, \xi(x)) \in \Gamma_{i}$ for each $x \in D_{i}$. The theorem now follows by partitioning further with respect to the $x$-variables and using the induction hypothesis.

Proof of Theorem 2.3. We only have to show that we can partition $X$ using analytic cells $A$ in such a way that the functions $\delta_{j}$ are analytic on $\pi_{m}(A)$. In [9] one proves that any subanalytic function is piecewise analytic. Theorem[2.3 then follows from Theorem 2.3 by partitioning further using this fact.

\section{Classification of subanalytic sets}

For $X \subset K^{m}$ subanalytic and nonempty, the dimension $\operatorname{dim}(X)$ of $X$ is defined as the largest integer $n$ such that there is a $K$-linear map $\pi: K^{m} \rightarrow K^{n}$ and a nonempty $U \subset \pi(X)$, open in $K^{n}$ (for alternative definitions, see [9]).

Theorem 3.1. For any subanalytic set $X \subset K^{m}$ and subanalytic functions $f_{i}$ : $X \rightarrow K, i=1, \ldots, r$, there is a semialgebraic set $Y$, a subanalytic bijection $F$ : $X \rightarrow Y$ and there are semialgebraic maps $g_{i}: Y \rightarrow K$ such that

$$
\left|g_{i}(F(x))\right|=\left|f_{i}(x)\right| \quad \text { for each } x \in X .
$$

Proof. We will give a proof by induction on $m$. Suppose that $X \subset K^{m+1}$ is subanalytic and that $f_{i}: X \rightarrow K$ are subanalytic functions, $m \geq 0$. Apply cell decomposition to $X$ and the functions $f_{i}$ to obtain a finite partition $\mathcal{P}$ of $X$. For $A \in \mathcal{P}$ and $(x, t) \in A$, suppose that $\left|f_{i}(x, t)\right|=\left|\delta_{i}(x)\right|\left|(t-\gamma(x))^{a_{i}} \mu^{-a_{i}}\right|^{\frac{1}{n}}, i=1, \ldots, r$, and suppose that $A$ is a cell of the form

$$
\left\{(x, t) \in K^{m+1}|x \in D,| \alpha(x)\left|\square_{1}\right| t-\gamma(x)\left|\square_{2}\right| \beta(x) \mid, t-\gamma(x) \in \mu P_{n}\right\},
$$

as in (3). After the translation $(x, t) \mapsto(x, t-\gamma(x))$ we may suppose that $\gamma$ is zero on $D$. Apply the induction hypotheses to the sets $D$ and the subanalytic functions $\alpha, \beta$, and $\delta_{i}$. Repeating this process for every $A \in \mathcal{P}$, and noting that there is a semialgebraic function $h: P_{n} \rightarrow K$ such that $|h(t)|=|t|^{1 / n}$, the proposition follows after taking appropriate disjoint unions inside $K^{m}$ of the occurring semialgebraic sets.

We prove the following generalization of Theorem 1.2

Theorem 3.2. Let $X \subset K^{m}$ and $Y \subset K^{n}$ be infinite subanalytic sets. Then there exists a subanalytic bijection $X \rightarrow Y$ if and only if $\operatorname{dim}(X)=\operatorname{dim}(Y)$. 
Proof. By Theorem 3.1 there are subanalytic bijections $X \rightarrow X^{\prime}$ and $Y \rightarrow Y^{\prime}$ with $X^{\prime}$ and $Y^{\prime}$ semialgebraic, but then there exists a semialgebraic bijection $X^{\prime} \rightarrow Y^{\prime}$ if and only if $\operatorname{dim}\left(X^{\prime}\right)=\operatorname{dim}\left(Y^{\prime}\right)$ by Theorem 2 of [2]. Since the dimension of a subanalytic set is invariant under subanalytic bijections (see [9]), the theorem follows.

\section{Parametrized analytic integrals}

We show that certain algebras of functions from $\mathbb{Q}_{p}^{m}$ to the rational numbers $\mathbb{Q}$ are closed under $p$-adic integration. These functions are called subanalytic constructible functions, and they come up naturally when one calculates parametrized $p$-adic integrals such as (1).

For $x=\left(x_{1}, \ldots, x_{m}\right)$ an $m$-tuple of variables, we will write $|d x|$ to denote the Haar measure on $K^{m}$, so normalized that $R^{m}$ has measure 1 .

Definition 4.1. For each subanalytic set $X$, we let $\mathcal{C}(X)$ be the $\mathbb{Q}$-algebra generated by the functions $X \rightarrow \mathbb{Q}$ of the form $x \mapsto v(h(x))$ and $x \mapsto\left|h^{\prime}(x)\right|$, where $h: X \rightarrow K^{\times}$and $h^{\prime}: X \rightarrow K$ are subanalytic functions. We call $f \in \mathcal{C}(X)$ a subanalytic constructible function on $X$.

To any function $f$ in $\mathcal{C}\left(K^{m+n}\right), m, n \geq 0$, we associate a function $I_{m}(f): K^{m} \rightarrow$ $\mathbb{Q}$ by putting

$$
I_{m}(f)(\lambda)=\int_{K^{n}} f(\lambda, x)|d x|
$$

if the function $x \mapsto f(\lambda, x)$ is absolutely integrable for all $\lambda \in K^{m}$, and we put $I_{m}(f)(\lambda)=0$ otherwise.

Theorem 4.2 (Basic Theorem on $p$-Adic Analytic Integrals). For any function $f \in \mathcal{C}\left(K^{m+n}\right)$, the function $I_{m}(f)$ is in $\mathcal{C}\left(K^{m}\right)$.

Proof. By induction it is enough to prove that for a function $f$ in $\mathcal{C}\left(K^{m+1}\right)$ in the variables $\left(\lambda_{1}, \ldots, \lambda_{m}, t\right)$ the function $I_{m}(f)$ is in $\mathcal{C}\left(K^{m}\right)$. Suppose that $f$ is a $\mathbb{Q}$ linear combination of products of functions $\left|f_{i}\right|$ and $v\left(g_{j}\right), i=1, \ldots, r, j=1, \ldots, s$, where $f_{i}$ and $g_{j}$ are subanalytic functions $K^{m+1} \rightarrow K$ and $g_{j}(\lambda, t) \neq 0$. Applying cell decomposition to $K^{m+1}$ and the functions $f_{i}$ and $g_{j}$, we obtain a partition $\mathcal{P}$ of $K^{m+1}$ into cells such that $I_{m}(f)(\lambda)$ is a sum of integrals over $A_{\lambda}=\{t \mid(\lambda, t) \in A\}$ for each cell $A \in \mathcal{P}$, where the integrands on these pieces $A_{\lambda}$ have a very simple form. More precisely, on each piece $A_{\lambda}$ the integrand is a $\mathbb{Q}$-linear combination of functions of the form

$$
\delta(\lambda)\left|(t-\gamma)^{a} \mu^{-a}\right|^{\frac{1}{n}} v(t-\gamma(\lambda))^{l},
$$

where $A$ is a cell with center $\gamma: K^{m} \rightarrow K$ and coset $\mu P_{n}$, and with integers $a$ and $0 \leq l$, and a function $\delta$ in $\mathcal{C}\left(K^{m}\right)$. We may suppose that $\delta(\lambda) \neq 0$ for some $\lambda \in \pi_{m}(A)$. Regroup all such terms where the same exponents $a$ and $l$ appear, possibly by replacing the functions $\delta(\lambda)$ by other functions in $\mathcal{C}\left(K^{m}\right)$. The integrability of such an integrand then only depends on the integers $a, l$, and $n$ occurring in each of the terms as in (6) and on the symbols $\square_{i}$ and $\mu$ occurring in the description of the cell $A$. By consequence, we may suppose that the integrand is a single term of the form as in (6) and that this term is absolutely integrable 
over $A$. It suffices to show that the integral

$$
\delta(\lambda) \int_{t \in A_{\lambda}}\left|(t-\gamma(\lambda))^{a} \mu^{-a}\right|^{\frac{1}{n}} v(t-\gamma(\lambda))^{l}|d t|
$$

is in $\mathcal{C}\left(K^{m}\right)$. Write $u=t-\gamma(\lambda)$; since $A$ is a cell with center $\gamma$ and coset $\mu P_{n}$, the set $A$ is of the form

$$
A=\left\{(\lambda, u) \in K^{m+1}|\lambda \in D,| \alpha(\lambda)\left|\square_{1}\right| u\left|\square_{2}\right| \beta(\lambda) \mid, u \in \mu P_{n}\right\},
$$

with $\square_{i}$ either $<$ or no condition, $D$ a cell, and $\alpha, \beta: K^{m} \rightarrow K^{\times}$subanalytic functions. Taking into account that the integral (7) is, by supposition, integrable, only a few possibilities can occur (with respect to the integers $a, l$, and $n$, the conditions $\square_{i}$, and $\mu$ being zero or nonzero). If $\mu=0$, the set $A_{\lambda}$ is a point for each $\lambda \in D$, thus the statement is clear. Suppose $\mu \neq 0$. In case that both $\square_{1}$ and $\square_{2}$ represent no condition, the integrand has to be zero by the supposition of integrability, and the above integral trivially is in $\mathcal{C}\left(K^{m}\right)$. We suppose from now on that $\square_{1}$ is $<$; the other cases can be treated similarly. The integral (7) can be rewritten as

$$
\begin{gathered}
\delta(\lambda) \cdot \int_{u \in A_{\lambda}}\left|u^{a} \mu^{-a}\right|^{\frac{1}{n}} v(u)^{l}|d u| \\
=\delta(\lambda) \sum_{k}\left(q^{-a k}\left|\mu^{-a}\right|\right)^{\frac{1}{n}} k^{l} \cdot \text { Measure }\left\{u \in A_{\lambda} \mid v(u)=k\right\} \\
=\epsilon \delta(\lambda) \sum_{k}\left(q^{-a k}\left|\mu^{-a}\right|\right)^{\frac{1}{n}} k^{l} q^{-k}
\end{gathered}
$$

for $\epsilon=q^{s} \cdot$ Measure $\left\{u \in A_{\lambda} \mid v(u)=s\right\}$ (where $s$ is any number such that $\emptyset \neq\{u \in$ $\left.A_{\lambda} \mid v(u)=s\right\}$ ), and where the summation is over those integers $k \equiv v(\mu) \bmod n$ satisfying

$$
|\alpha(\lambda)|<q^{-k} \square_{2}|\beta(\lambda)| \text {. }
$$

We may suppose that on $A$, the residue classes

$$
v(\alpha(\lambda)) \quad(\bmod n) \text { and } v(\beta(\lambda)) \quad(\bmod n)
$$

are fixed (possibly after refining the partition $\mathcal{P}$ ). Then this sum is equal to a $\mathbb{Q}$-linear combination of products of the functions $\delta,|\alpha|,|\beta|, v(\alpha)$ and $v(\beta)$. For example, if $a / n=-1, \square_{1}$ and $\square_{2}$ are necessarily $<$ and one obtains a polynomial in $v(\alpha)$ and $v(\beta)$ of degree $\leq l+1$, multiplied with $\delta$. For more examples of calculations of sums of this kind, see [5], proof of Lemma 3.2. Thus, the integral (7) is in $\mathcal{C}\left(K^{m}\right)$ as was to be shown.

As a corollary we will formulate another version of the basic integration theorem, conjectured in [8] in the remark following Theorem 2.6.

Definition 4.3. A set $A \subset \mathbb{Z}^{n} \times K^{m}$ is called simple if

$$
\left\{(\lambda, x) \in K^{n+m} \mid\left(v\left(\lambda_{1}\right), \ldots, v\left(\lambda_{n}\right), x\right) \in A \& \prod_{i=1 \ldots, n} \lambda_{i} \neq 0\right\}
$$

is a subanalytic set. A function $h: A \subset \mathbb{Z}^{n} \times \mathbb{Q}_{p}^{m} \rightarrow \mathbb{Z}$ is called simple if its graph is simple. For a simple set $X$ we let $\mathcal{C}_{\text {simple }}(X)$ be the $\mathbb{Q}$-algebra generated by all simple functions on $X$ and all functions of the form $q^{h}$, where $h$ is a simple function on $X$. 
For a function $f$ in $\mathcal{C}_{\text {simple }}\left(\mathbb{Z}^{k+l} \times K^{m+n}\right), k, l, m, n$ integers $\geq 0$, we define $I_{k, m}(f): \mathbb{Z}^{k} \times K^{m} \rightarrow \mathbb{Q}$ as

$$
I_{k, m}(f)(z, \lambda)=\sum_{z^{\prime} \in \mathbb{Z}^{l}} \int_{K^{n}} f\left(z, z^{\prime}, \lambda, x\right)|d x|
$$

if the function $\left(z^{\prime}, x\right) \mapsto f\left(z, z^{\prime}, \lambda, x\right)$ is absolutely integrable for all $(z, \lambda) \in \mathbb{Z}^{k} \times K^{m}$ with respect to the Haar measure on $K^{n}$ and the discrete measure on $\mathbb{Z}^{l}$, and we define $I_{k, m}(f)(z, \lambda)=0$ otherwise.

Theorem 4.4. For each $f$ in $\mathcal{C}_{\text {simple }}\left(\mathbb{Z}^{k+l} \times K^{m+n}\right)$, the function $I_{k, m}(f)$ is in $\mathcal{C}_{\text {simple }}\left(\mathbb{Z}^{k} \times K^{l}\right)$.

Proof. It is enough to prove that for a function $f$ in $\mathcal{C}_{\text {simple }}\left(\mathbb{Z}^{k} \times K^{m}\right)$ in the variables $\left(z_{1}, \ldots, z_{k}, x_{1}, \ldots, x_{m}\right)$ the function obtained by eliminating $x_{m}$ by integration, resp. eliminating $z_{k}$ by summation, is in the respective algebra $\mathcal{C}_{\text {simple. }}$.

We first focus on integration with respect to $x_{m}$. To $f: \mathbb{Z}^{k} \times K^{m} \rightarrow \mathbb{Q}$ we can associate a function $g: K^{k+m} \rightarrow \mathbb{Q}$ by replacing the variables $z$ running over $\mathbb{Z}^{k}$ by variables $\lambda$ running over $K^{k}$ in such a way that $g(\lambda, x)=f\left(v\left(\lambda_{1}\right), \ldots, v\left(\lambda_{k}\right), x\right)$ for each $\lambda \in\left(K^{\times}\right)^{k}$ and $g(\lambda, x)=0$ if one of the $\lambda_{i}$ is zero. By the definitions it is immediate that $g$ is in $\mathcal{C}\left(K^{k+m}\right)$ and the integral of $f$ with respect to $x_{m}$ corresponds to the integral of the function $g$ with respect to $x_{m}$. If we eliminate $x_{m}$ by integration from $g$, then we get the function $I_{k+m-1}(g)$ which is in $\mathcal{C}\left(K^{k+m-1}\right)$ by Theorem 4.2. This function only depends on $\left(v\left(\lambda_{1}\right), \ldots, v\left(\lambda_{k}\right), x_{1}, \ldots, x_{m-1}\right)$ and thus corresponds to a function in $\mathcal{C}_{\text {simple }}\left(K^{k+m-1}\right)$, as one can check.

If we want to eliminate $z_{k}$ by summation, we associate to $f$ the subanalytic constructible function $g^{\prime}: K^{k+m} \rightarrow \mathbb{Q}$ determined by

$$
g^{\prime}(\lambda, x)=\left|\lambda_{k}\right|^{-1} \frac{p}{p-1} f\left(v\left(\lambda_{1}\right), \ldots, v\left(\lambda_{k}\right), x\right)
$$

if $\prod_{i=1 \ldots, n} \lambda_{i} \neq 0$ and $g^{\prime}(\lambda, x)=0$ if $\prod_{i=1 \ldots, n} \lambda_{i}=0$. Integrating with respect to $\lambda_{k}$ then corresponds to summing over $z_{k}$, and the same argument as above can be applied to complete the proof.

\section{ACKNOWLEDGMENT}

I thank Denef for pointing out to me his conjecture on $p$-adic subanalytic integrals and for having stimulating conversations on this and related subjects. I thank van den Dries, Haskell, and Mourgues for many interesting discussions. I also thank the referee for his useful suggestions on the presentation of the paper.

\section{REFERENCES}

1. N. Bourbaki, Variétés différentielles et analytiques. Fascicule de résultats, Hermann, Paris, 1967, (French). MR 36:2161

2. R. Cluckers, Classification of semialgebraic p-adic sets up to semialgebraic bijection, Journal für die reine und angewandte Mathematik 540 (2001), 105-114. MR 2002i:14052

3. R. Cluckers and D. Haskell, Grothendieck rings of $\mathbb{Z}$-valued fields, Bulletin of Symbolic Logic 7 (2001), no. 2, 262-269. MR 2002g:03081

4. J. Denef, The rationality of the Poincaré series associated to the p-adic points on a variety, Inventiones Mathematicae 77 (1984), 1-23. MR 86c:11043

5. - On the evaluation of certain p-adic integrals, Théorie des nombres, Sémin. DelangePisot-Poitou 1983-84, vol. 59, 1985, pp. 25-47. 
6. __ p-adic semialgebraic sets and cell decomposition, Journal für die reine und angewandte Mathematik 369 (1986), 154-166. MR 88d:11030

7. Multiplicity of the poles of the Poincaré series of a p-adic subanalytic set, Sém. Th. Nombres Bordeaux 43 (1987-1988), 1-8. MR 90f:11089

8. - Arithmetic and geometric applications of quantifier elimination for valued fields, MSRI Publications, vol. 39, pp. 173-198, Cambridge University Press, 2000. MR 2001e:03063

9. J. Denef and L. van den Dries, $p$-adic and real subanalytic sets, Annals of Mathematics 128 (1988), no. 1, 79-138. MR 89k:03034

10. J. Denef and F. Loeser, Germs of arcs on singular algebraic varieties and motivic integration, Inventiones Mathematicae 135 (1999), 201-232. MR 99k:14002

11. _ Definable sets, motives and p-adic integrals, Journal of the American Mathematical Society 14 (2001), no. 2, 429-469. MR 2002k:14033

12. L. van den Dries, Tame topology and o-minimal structures, Lecture note series, vol. 248, Cambridge University Press, 1998. MR 99j:03001

13. L. van den Dries, D. Haskell, and D. Macpherson, One-dimensional p-adic subanalytic sets, Journal of the London Mathematical Society 59 (1999), no. 1, 1-20. MR 2000k:03077

14. M.P.F. du Sautoy, Finitely generated groups, p-adic analytic groups and Poincaré series, Annals of Mathematics 137 (1993), no. 3, 639-670. MR 94j:20029

15. W. Hodges, Model theory, Encyclopedia of Mathematics and Its Applications, vol. 42, Cambridge University Press, 1993. MR 94e:03002

16. J. Igusa, Complex powers and asymptotic expansions I, Journal für die reine und angewandte Mathematik 268 (1974), 110-130. MR 50:254

17. Complex powers and asymptotic expansions II, Journal für die reine und angewandte Mathematik 278 (1975), 307-321. MR 53:8018

18. Lectures on forms of higher degree (notes by S. Raghavan), Lectures on mathematics and physics, Tata Institute of Fundamental Research, vol. 59, Springer-Verlag, 1978. MR 80m:10020

19. B. Lichtin, On a question of Igusa: towards a theory of several variable asymptotic expansions I, Compositio Mathematica 120 (2000), no. 1, 25-82. MR 2001c:11086

20. J.-M. Lion and J.-P. Rolin, Intégration des fonctions sous-analytiques et volumes des sousensembles sous-analytiques (Integration of subanalytic functions and volumes of subanalytic subspaces), Ann. Inst. Fourier 48 (1998), no. 3, 755-767 (French). MR 2000i:32011

21. L. Lipshitz and Z. Robinson, Rings of separated power series and quasi-affinoid geometry, Paris: Société Mathématique de France, vol. 264, Astérisque, 2000. MR 2001g:32017

22. Nianzheng Liu, Analytic cell decomposition and the closure of p-adic semianalytic sets, Journal of Symbolic Logic 62 (1997), no. 1, 285-303. MR 98h:03039

23. A. Macintyre, On definable subsets of p-adic fields, Journal of Symbolic Logic 41 (1976), 605-610. MR 58:5182

24. M.-H. Mourgues, Corps p-minimaux avec fonctions de Skolem définissables, Séminaire de structures algébriques ordonnées, 1999-2000, prépublication de l'équipe de logique mathématique de Paris 7, pp. 1-8.

25. A. Mylnikov, p-adic subanalytic preparation and cell decomposition theorems, Ph.D. thesis, Purdue University, 1999.

26. J. Oesterlé, Réduction modulo $p^{n}$ des sous-ensembles analytiques fermés de $\mathbb{Z}_{p}^{n}$, Inventiones Mathematicae 66 (1982), no. 2, 325-341. MR 83j:12014

27. J.-P. Serre, Quelques applications du théorème de densité de Chebotarev, Publ. Math. Inst. Hautes Études Sci. 323-401 (1981). MR 83k:12011

28. S. Wilcox, Topics in the model theory of p-adic numbers, Ph.D. thesis, University of Oxford, (unfinished).

Department of Mathematics, Katholieke Universiteit Leuven, Celestijnenlaan 200B, 3001 Leuven, Belgium

E-mail address: raf.cluckers@wis.kuleuven.ac.be

$U R L:$ http://www.wis.kuleuven.ac.be/wis/algebra/Raf/index.html

Current address: École Normale Supérieure, Département de Mathématiques et Applications, 45 rue d'Ulm, 75230 Paris Cedex 05, France 